\title{
A Transverse Oscillation Approach for Estimation of Three-Dimensional Velocity Vectors, Part II: Experimental Validation
}

Pihl, Michael Johannes; Stuart, Matthias Bo; Tomov, Borislav Gueorguiev; Rasmussen, Morten Fischer; Jensen, Jørgen Arendt

Published in:

IEEE Transactions on Ultrasonics, Ferroelectrics and Frequency Control

Link to article, DOI:

10.1109/TUFF c .2013.006238

Publication date:

2014

Link back to DTU Orbit

Citation (APA):

Pihl, M. J., Stuart, M. B., Tomov, B. G., Rasmussen, M. F., \& Jensen, J. A. (2014). A Transverse Oscillation Approach for Estimation of Three-Dimensional Velocity Vectors, Part II: Experimental Validation. IEEE Transactions on Ultrasonics, Ferroelectrics and Frequency Control, 61(10), 1608-1618. https://doi.org/10.1109/TUFF c .2013.006238

\section{General rights}

Copyright and moral rights for the publications made accessible in the public portal are retained by the authors and/or other copyright owners and it is a condition of accessing publications that users recognise and abide by the legal requirements associated with these rights.

- Users may download and print one copy of any publication from the public portal for the purpose of private study or research.

- You may not further distribute the material or use it for any profit-making activity or commercial gain

- You may freely distribute the URL identifying the publication in the public portal 


\title{
A Transverse Oscillation Approach for Estimation of Three-Dimensional Velocity Vectors, Part II: Experimental Validation
}

\author{
Michael Johannes Pihl, Member, IEEE, Matthias Bo Stuart, Borislav Gueorguiev Tomov, \\ Morten Fischer Rasmussen, and Jørgen Arendt Jensen, Fellow, IEEE
}

\begin{abstract}
The 3-D transverse oscillation method is investigated by estimating 3-D velocities in an experimental flow-rig system. Measurements of the synthesized transverse oscillating fields are presented as well. The method employs a 2-D transducer; decouples the velocity estimation; and estimates the axial, transverse, and elevation velocity components simultaneously. Data are acquired using a research ultrasound scanner. The velocity measurements are conducted with steady flow in sixteen different directions. For a specific flow direction with $[\alpha, \beta]$ $=[45,15]^{\circ}$, the mean estimated velocity vector at the center of the vessel is $\left(v_{x}, v_{y}, v_{z}\right)=(33.8,34.5,15.2) \pm(4.6,5.0,0.6) \mathrm{cm} / \mathrm{s}$ where the expected velocity is $(34.2,34.2,13.0) \mathrm{cm} / \mathrm{s}$. The velocity magnitude is $50.6 \pm 5.2 \mathrm{~cm} / \mathrm{s}$ with a bias of $0.7 \mathrm{~cm} / \mathrm{s}$. The flow angles $\alpha$ and $\beta$ are estimated as $45.6 \pm 4.9^{\circ}$ and 17.6 $\pm 1.0^{\circ}$. Subsequently, the precision and accuracy are calculated over the entire velocity profiles. On average for all direction, the relative mean bias of the velocity magnitude is $-0.08 \%$. For $\alpha$ and $\beta$, the mean bias is $-0.2^{\circ}$ and $-1.5^{\circ}$. The relative standard deviations of the velocity magnitude ranges from 8 to $16 \%$. For the flow angles, the ranges of the mean angular deviations are $5^{\circ}$ to $16^{\circ}$ and $0.7^{\circ}$ and $8^{\circ}$.
\end{abstract}

\section{INTRODUCTION}

$\mathrm{U}$ LTRASONIC velocity estimation has come far since the first measurements were conducted [1], but the conventional methods in commercial scanners estimate only the axial velocity component. However, this does not capture the blood flow in all its complexity [2]-[4]. Techniques that are able to estimate all three directional components, and thereby the correct velocity magnitudes directly, will change the diagnostic value and reduce the work load for physicians or sonographers. In addition, visualizations of complex flow patterns around stenoses, valves, etc. will provide new quantitative data on the hemodynamics and contribute to the diagnostic process. For example, it will become possible to estimate true volumetric flow rates without assumptions on vessel geometry.

Over the past decades, several authors have presented results of measured true or pseudo three-dimensional (3D) velocities. The published results have been based on a range of techniques, including cross-beam methods [5]-[7],

Manuscript received December 17, 2013; accepted April 20, 2014. This work was supported by grant 024-2008-3 from the Danish Advanced Technology Foundation and BK Medical ApS, Denmark.

The authors are with the Center for Fast Ultrasound Imaging, Department of Electrical Engineering, Technical University of Denmark, Kgs. Lyngby, Denmark (e-mail: jaj@elektro.dtu.dk).

DOI http://dx.doi.org/10.1109/TUFFC.2013.006238 speckle tracking [8], feature tracking [9], transverse Doppler methods using the spectral broadening effect [10], decorrelation based techniques [11], [12], cross-correlation of beams [13], [14], and particle imaging velocimetry [15]. The proposed techniques have various limitations such as limited field of view, high computational demands, or the need for contrast agents. So far, none of these techniques have produced in vivo 3-D vector flow images and none of them have been adopted by commercial manufactures.

The authors have suggested the 3 -D transverse oscillation (TO) method for estimating 3-D velocity vectors by means of two pairs of double-oscillating fields, spatial quadrature sampling, and the use of a 2-D transducer array. The method is presented in the companion paper [16], where simulations demonstrate the ability of the 3-D TO method to estimate 3 -D velocity vector profiles.

This paper presents estimated 3-D vector velocities based on measurements conducted in a steady flow-rig system using the 3-D TO method. The purpose is to experimentally verify the applicability of the method. This is achieved by measuring the transverse oscillating fields and by measuring 3-D velocities through a rubber vessel in a flow-rig system using an experimental research scanner and a 2-D transducer array. Measurements are conducted for sixteen different flow angles.

Section II briefly refers to the 3-D transverse oscillation approach. The analysis of spherical data follows in Section III. Section IV describes the experimental setup, and the results are presented and discussed in Sections V and VI. The conclusions are stated in Section VII.

\section{The Transverse Oscillation Method}

The 3-D TO method is presented in the companion paper [16], which describes the concept, the beamforming, and the velocity estimation based on simulated data. This paper presents the use of that method for estimating 3-D velocities from experimentally acquired data.

The originally proposed TO method for $2-\mathrm{D}$ velocity estimation employed linear-array transducers, and experimentally obtained in vivo results using the TO method for 2-D vector velocity imaging have been presented [17]. The 2-D method has been implemented on a commercial scanner and FDA approved for clinical use, and clinical in vivo 2 -D vector flow images obtained using the commercial scanner have been demonstrated [18], [19]. The 3-D 
TO method expands that functionality for achieving 3-D velocity vector imaging.

\section{Analysis of Spherical Data}

The spherical coordinates system used to define the different flow directions in 3-D space is described in the following. Subsequently, the angle estimation and the statistics methods used to calculate the mean and standard deviation of angular data are described.

\section{A. Spherical Coordinate System}

The flow direction is defined by two angles, $\alpha$ and $\beta$. For a given vector, the angle $\alpha$ is defined as the angle in the $X Y$-plane defined by the transducer as illustrated in Fig. 1 . The angle $\beta$ is between the $X Y$-plane and the vector. Thus, $-180^{\circ} \leq \alpha<180^{\circ}$ and $-90^{\circ} \leq \beta<90^{\circ}$.

Thereby, $\alpha$ and $\beta$ correspond to longitude and latitude in the geographical coordinate system [20]. Compared with the polar coordinates $(\theta$ and $\phi)$, the following relations exist:

$$
\theta=90^{\circ}-\beta, \quad \phi=\alpha .
$$

\section{B. Angle Estimation}

The 3-D method estimates the velocity components $v_{x}$, $v_{y}$, and $v_{z}$. Hence, the angles $\alpha$ and $\beta$ for each estimate are

$$
\begin{aligned}
& \alpha=\arctan \frac{v_{y} /\|v\|}{v_{x} /\|v\|}=\arctan \frac{v_{y}}{v_{x}} \\
& \beta=\sin \frac{v_{z}}{\|v\|},
\end{aligned}
$$

where the velocity components have been normalized by the velocity magnitude $\|v\|$. Note that the arctan operator should be the four-quadrant inverse tangent operator to get the full angular range.

\section{Circular and Spherical Descriptive Statistics}

To calculate the mean and standard deviation of the velocity estimates on angular scales, appropriate methods must be applied that differ from standard linear statistics [20], [21].

For the estimation of the mean and standard deviation (angular deviation) of $\alpha$ and $\beta$, circular statistics was employed on the two angles separately using the Matlab (MathWorks, Inc., Natick, MA) toolbox CircStat: A Matlab Toolbox for Circular Statistics [21]. Note that the angular deviations are calculated for $\alpha$ and $\beta$ separately, and hence do not describe the interaction of the two.

To estimate and visualize the mean direction depending on both $\alpha$ and $\beta$, spherical statistics [20] were applied. An in-house modified version of the Spak software

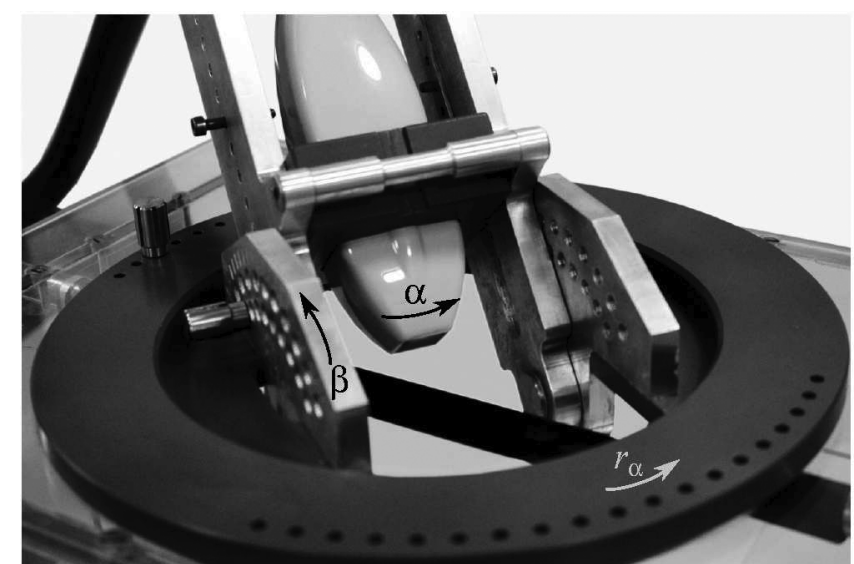

Fig. 1. The 2-D ultrasound probe positioned relative to the rubber tube in the flow rig by a fixture. A desired beam-to-flow orientation can be obtained by adjusting the two angles $\alpha$ and $\beta$. When $\beta=0^{\circ}, \alpha$ can be obtained by rotating $r_{\alpha}$.

(implemented in Matlab) by Leong and Carlile [22] was employed.

\section{Experimental Setup}

This section describes the equipment and methods used to obtain the experimental data. Two types of experiments were conducted. First, measurements of the TO fields using a scanning tank system were performed. The purpose of that measurement was to verify that the spatial in-phase and quadrature (IQ) sampling approach works as expected. Next, velocity measurements in an inhouse-built flow-rig system were carried out to demonstrate the feasibility of using the 3-D TO method for 3-D velocity vector estimation.

The following describes the measurement equipment, the measurement setup, the data acquisition, and the data processing.

\section{A. Measurement Equipment}

The main requirement for experimentally measuring the TO fields and 3-D vector velocities is a $2-\mathrm{D}$ transducer array and a system that can sample and store the echo signals for every transducer element. Both will be introduced subsequently, followed by a description of the scanning tank system used for measuring the TO fields and the flow-rig system used for velocity measurements.

The ultrasound probe used was a 2-D transducer array fabricated by Vermon S.A., Tours, France [23], [24] as shown in Fig. 1. The transducer has $32 \times 32$ active elements with a pitch in both dimensions of $0.3 \mathrm{~mm}$. The center frequency of the transducer is $3.5 \mathrm{MHz}$ and the two-way impulse response has a bandwidth of $67 \%$.

The 1024 active elements on the transducer are connected to the 1024 channels on the experimental scanner SARUS [25]. The signals on all 1024 channels were 
sampled simultaneously and synchronously at a frequency of $70 \mathrm{MHz}$.

Besides sampling the echo signals from transducers, SARUS can sample signals from a hydrophone. An HGL0400 capsule hydrophone (Onda Corp., Sunnyvale, CA) was mounted in an AIMS III scanning tank (Onda Corp.), which controls the position of the hydrophone in all three spatial dimensions. The hydrophone is connected through a preamplifier to either an oscilloscope or to SARUS. SARUS and the scanning tank system were controlled from the same Matlab program to ensure synchronized data acquisition.

An in-house-built flow-rig system was used for the experimental velocity measurements. It is a closed-loop circuit, and consists of a Cole-Parmer (Vernon Hills, IL) centrifugal pump, an airtrap, a 1.2-m metal tube, followed by a rubber (heat-shrink material) tube penetrable to ultrasound inside a tank filled with demineralized water. The length of the metal tube is long enough to ensure fully developed laminar flow, which has a parabolic flow profile. The internal radius $r$ of both the metal and rubber tubes is $6 \mathrm{~mm}$, and the wall thickness of the rubber tube is $0.5 \mathrm{~mm}$. The centrifugal pump circulates a bloodmimicking fluid [26] manufactured by Danish Phantom Design (Frederikssund, Denmark). An industrial grade, calibrated magnetic MAG1100 flowmeter (Danfoss, Nordborg, Denmark) measures the volumetric flow rate, $Q$, with a systematic accuracy of $0.5 \%$.

Prior to measuring, an ultrasound probe fixture can be lowered into the water tank at adjustable beam-toflow angles (see Fig. 1). As the probe holder allowed some undesired tilt around the $x$ and $y$ axis, the overall uncertainty of the angles $\alpha$ and $\beta$ was approximately 1 to $2^{\circ}$.

\section{B. Measurement Setup and Data Acquisition}

The parameters for transmit and receive and their corresponding values used for measurements of the transverse oscillating fields and the 3 -D velocities are listed in Table I. The apodization parameters were chosen based on the parameter study in the companion paper [16].

For validation of the 3 -D TO spatial quadrature approach, the TO fields were investigated through pulseecho measurements in the scanning tank system, where the hydrophone was used as a point target. The TO fields were sampled at points forming a cross with the center at $[x, y, z]=[0,0,30] \mathrm{mm}$ and points along the $x$-axis and the $y$-axis. The transducer was centered at $[x, y, z]=$ $[0,0,0] \mathrm{mm}$. For each position of the point target, an excitation waveform was emitted and the signals from all 1024 channels were sampled. Subsequently, the same four TO lines were beamformed simultaneously for each position to obtain the TO fields. For these measurements, plane waves were emitted instead of a focused beam.

Velocity measurements were performed in the flow-rig system, which is assumed to have a steady, fully developed 2-D circular symmetric parabolic profile as illustrated in Fig. 2. Using the volumetric flow rate measured using the

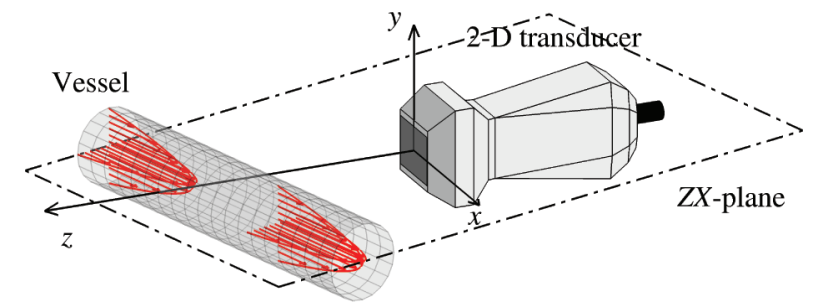

Fig. 2. Illustration of the flow phantom used for measurements. Here, the flow angle $\alpha$ is $0^{\circ}$ and $\beta$ is $-15^{\circ}$.

magnetic flowmeter and considering the geometry of the vessel, the peak velocity $v_{0}$ can be estimated. The pump was adjusted to obtain an expected peak velocity of approximately $0.5 \mathrm{~m} / \mathrm{s}$, because the pump cannot deliver a steady flow at high velocities. At this velocity, the Reynolds number is 775 . Considering transducer and vessel orientations, the three expected velocity components profiles were determined. They are parabolic in shape because the flow is assumed to be fully developed, and their width and maximum velocity depend on the beam-to-flow angles.

The transducer was positioned so that the center of the vessel was located at an axial depth of approximately $30 \mathrm{~mm}$. The transducer orientation can be chosen to obtain various combinations of the flow angles $\alpha$ and $\beta$. The flow angle $\alpha$ determines the relative magnitudes between $v_{x}$ and $v_{y}$, whereas $\beta$ is a tilt that determines the magnitude of the axial velocity component (see Figs. 1 and 2). For $[\alpha, \beta]=[0,0]^{\circ}$, the flow is fully in the $x$-direction, i.e., $(x, y, z)=(1,0,0)$. To align the flow direction to be fully in the $y$-direction, $[\alpha, \beta]$ should be $[90,0]^{\circ}$. If $[\alpha, \beta]=[45,45]^{\circ}$, the flow direction is $(1 / 2,1 / 2,1 / \sqrt{2})$.

Measurements were conducted at the following combinations of flow angles: with $\beta$ at either $0^{\circ}$ or $15^{\circ}, \alpha$ was varied from $0^{\circ}$ to $90^{\circ}$ in steps of $15^{\circ}$. Additional measurements were conducted with $\alpha$ at $45^{\circ}$ and $\beta$ at $30^{\circ}$ and $45^{\circ}$. Because of setup limitations, $\beta$ could not be larger than $45^{\circ}$.

The flow angles were obtained in the following way: when $\beta=0^{\circ}, \alpha$ was obtained by rotating $r_{\alpha}$ as illustrated in Fig. 1. This also worked as an approximation when $\beta$

Table i. Default Parameter Settings for the Measurements.

\begin{tabular}{ll}
\hline Parameter & Value \\
\hline Emitted pulse & \\
Center frequency [MHz] & 3.5 \\
Number of cycles & 8 \\
Windowing of emitted pulse & Hanning \\
Focal depth [mm] & 30 \\
Steering angle in azimuth plane, $\theta_{z x}\left[^{\circ}\right]$ & 0 \\
Steering angle in elevation plane, $\theta_{z y}\left[^{\circ}\right]$ & 0 \\
Transmit apodization & \\
Transmit apodization & Hamming $(32 \times 32)$ \\
Receive apodization & Hanning $(32 \times 32)$ \\
Center line & Rectangular \\
TO peak apod. (osc. direction) & Rectangular \\
TO peak apod. (non-osc. direction) & 24 \\
TO peak spacing $d[\mathrm{elements}]$ & 8 \\
TO window width [elements] &
\end{tabular}


$=15^{\circ}$. However, when $\beta$ was increased further, a second probe holder (with $\alpha=45^{\circ}$ ) had to be used.

For each flow direction, 3200 pulse-echo measurements were acquired. Depending on the flow orientation, the pulse repetition frequency, $f_{\text {prf }}$, had to be adjusted to avoid aliasing. At $\beta=0^{\circ}$, it is determined by the transverse velocity components and $f_{\text {prf }}$ was set to $900 \mathrm{~Hz}$ to have the maximum transverse velocity component at $67 \%$ of the aliasing limit. For $\beta \geq 15^{\circ}$, the axial velocity component is the limiting factor, and $f_{\text {prf }}$ was set to $[1530,2950,3750] \mathrm{Hz}$ for $\beta=[15,30,45]^{\circ}$, respectively, to keep the maximum axial velocity at $80 \%$ to $90 \%$ of the aliasing limit.

\section{Data Processing}

The data processing was performed offline. The raw channel data from the measurements were match-filtered by convolving with the time-reversed emitted pulse. The subsequent beamforming was carried out using Beamformation Toolbox 3 (BFT 3) [27].

Before the velocity estimation was performed, stationary echo filtering was applied by subtracting the mean ensemble value of $N_{i}$ emissions. The ensemble length in the velocity estimation was 32 . To improve the performance of the velocity estimates, the mean transverse wavelength, $\bar{\lambda}_{x}$, and the mean elevation wavelength, $\bar{\lambda}_{y}$, were used in both the beamforming stage and in the velocity estimation as opposed to using the theoretically derived wavelengths. This lowers the standard deviation and reduces the bias [16], [28]. The estimated mean values were determined from Field II [29], [30] simulations using the same parameter settings as in the experimental setup.

\section{Transverse Oscillating Fields}

The TO method depends on creating the two pairs of double-oscillating fields, where the oscillations are in the axial and the transverse direction, but not in the elevation direction, and conversely, where the oscillations are in the axial and the elevation direction, but not in the transverse direction.

Fig. 3 presents pulse-echo measurements of the TO fields for a specific time instance in the sampled signals. The point target was positioned at a depth of $30 \mathrm{~mm}$. Fig. $3(\mathrm{a})$ is for the left and right beams in the $Z X$-plane when sampling along the $x$-direction. Ideally, the right beam should be identical to the Hilbert transform of the left beam for the spatial IQ modulation to work perfectly. The same should be the case in Fig. 3(d), where the samples from the two TO beams in the $Z Y$-plane were obtained sampling along the $y$-direction.

The opposite should be the case in Figs. 3(b) and 3(c), where the signals from the left and right beams should be in phase because the TO fields were sampled in the nonoscillating direction.

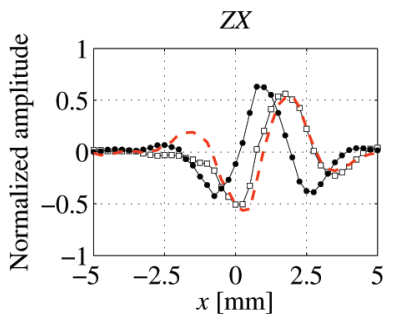

(a)

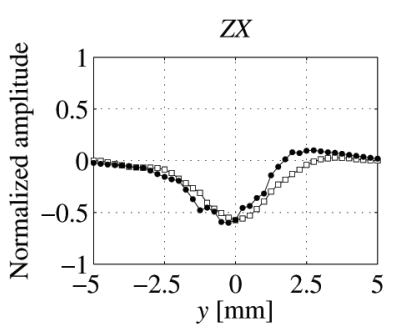

(c)

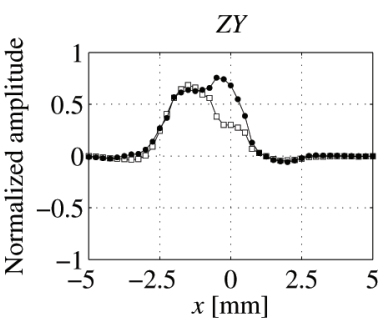

(b)

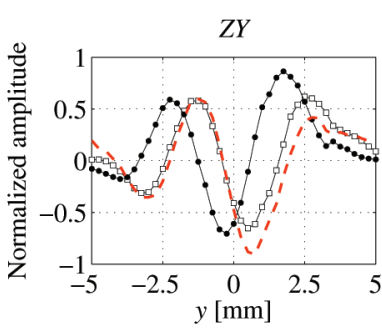

(d)
Fig. 3. Measured TO fields at a depth of $30 \mathrm{~mm}$ for a fixed time across either the transverse ( $a$ and $b$ ) or the elevation ( $c$ and $d)$. Dots and squares denote samples for the left and right beam, respectively. (a and c) are for the two beams in the $Z X$-plane. (b and d) are for the beams in the $Z Y$-plane. The dashed line is the Hilbert transform of the left beam (in a and $\mathrm{d}$ ).

The measured fields exhibit the expected trends, and a comparison with simulated results [31] using the same parameter settings shows comparable results.

The measured TO signals demonstrate that the 3-D spatial IQ approach works in an experimental setup, and that the TO fields oscillate only in either the transverse or the elevation direction.

\section{Measurements of 3-D Velocities}

M-mode flow measurements were conducted at sixteen different flow directions. The velocity components $v_{x}, v_{y}$, and $v_{z}$ along the center line were estimated using the 3-D TO method. For each measurement, 100 velocity profiles were estimated.

To provide an overview, the estimated mean $3-\mathrm{D}$ velocity vector at the center of the vessel for all measurements are visualized in Fig. 4 . The velocity vectors are normalized by their length and are illustrated as arrows within the unit sphere.

The results are analyzed in terms of their precision and accuracy. The order of presentation is as follows: First, the estimated 3-D velocity vectors at the center of the vessel are presented. Second, the corresponding velocity magnitudes and flow angles are calculated. Subsequently, the entire 3-D velocity profiles are investigated, followed by the calculated velocity magnitude and flow angle profiles. Finally, an alternative visualization of the mean flow directions is presented. 


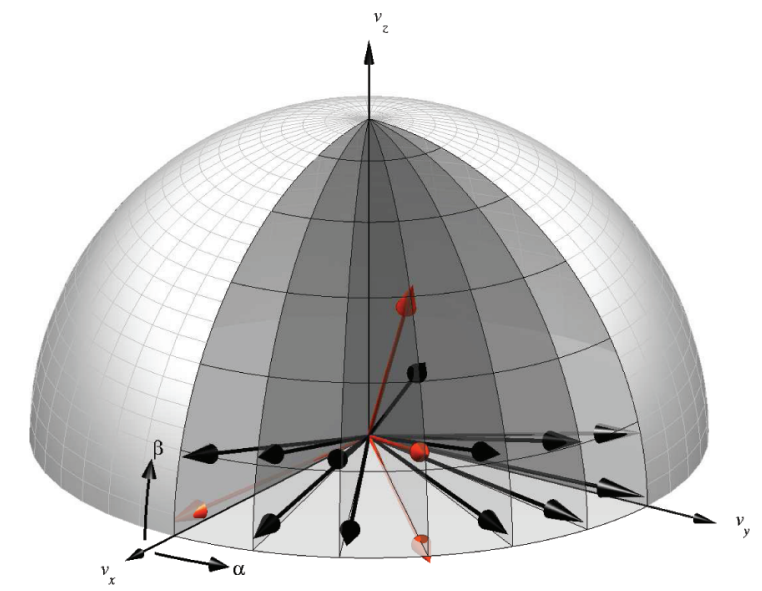

Fig. 4. The arrows represent the mean of the estimated 3-D velocity vectors at the center of the vessel for the 16 measurements conducted at various flow directions. The velocity vectors have been normalized with the peak velocity of $0.5 \mathrm{~m} / \mathrm{s}$. The non-black arrows correspond to the velocity profiles shown in Fig. 5. Ideally, the arrows should touch the intersections of the thin lines.

\section{A. 3-D Velocity Vectors at Center of Vessel}

The estimated velocity components for two of the velocity vectors shown in Fig. 4 are listed subsequently. For the flow direction $[\alpha, \beta]=[0,0]^{\circ}$, the mean and standard deviation of the velocity components at the center of the vessels are

$$
\overline{\mathbf{v}}=\left(\begin{array}{l}
\bar{v}_{x} \\
\bar{v}_{y} \\
\bar{v}_{z}
\end{array}\right)=\left(\begin{array}{r}
49.4 \\
-1.1 \\
1.7
\end{array}\right) \pm\left(\begin{array}{l}
3.2 \\
4.1 \\
0.4
\end{array}\right) \mathrm{cm} / \mathrm{s}
$$

where $\left(v_{x}, v_{y}, v_{z}\right)=(50,0,0) \mathrm{cm} / \mathrm{s}$ is the expected velocity. Normalized with the velocity magnitude, this yields relative bias magnitudes smaller than $4 \%$. The relative standard deviations are $6 \%, 8 \%$, and $0.9 \%$, respectively. The sizes of the components $v_{y}$ and $v_{z}$ indicate that $[\alpha, \beta]$ $\approx[1,2]^{\circ}$, and not $[\alpha, \beta] \approx[0,0]^{\circ}$ as expected, because of alignment uncertainties.

For the flow direction $[\alpha, \beta] \approx[45,15]^{\circ}$, the mean velocity vector is

$$
\overline{\mathbf{v}}=\left(\begin{array}{l}
\bar{v}_{x} \\
\bar{v}_{y} \\
\bar{v}_{z}
\end{array}\right)=\left(\begin{array}{l}
33.8 \\
34.5 \\
15.2
\end{array}\right) \pm\left(\begin{array}{l}
4.5 \\
5.0 \\
0.6
\end{array}\right) \mathrm{cm} / \mathrm{s}
$$

compared with the expected $\left(v_{x}, v_{y}, v_{z}\right)=$ $(34.2,34.2,13.0) \mathrm{cm} / \mathrm{s}$. This yields normalized biases of $-0.9 \%, 0.6 \%$, and $4 \%$ for $v_{x}, v_{y}$, and $v_{z}$. The relative standard deviations are $9 \%, 10 \%$, and 1\%, respectively.

The results demonstrate that it is possible to measure the $3-\mathrm{D}$ velocity vectors at the center of the vessel.

\section{B. Magnitude and Direction of 3-D Velocity Vectors}

Based on the three estimated velocity components, the velocity magnitude and direction can be estimated. The flow direction is described by the two flow angles $\alpha$ and $\beta$.

The means and standard deviations for four of the 16 flow directions are listed in Table II. In all four cases, the bias of the velocity magnitude is less than $2.4 \mathrm{~cm} / \mathrm{s}(5 \%)$. The bias of $\alpha$ is less than $2^{\circ}$ (out of $360^{\circ}$ ) and it is less than $3^{\circ}$ (out of $180^{\circ}$ ) for $\beta$. Because the estimation of the flow angles is sensitive to transducer orientation, the observed biases may arise from setup misalignment.

The standard deviation ranges from $3.2 \mathrm{~cm} / \mathrm{s}$ to $5.2 \mathrm{~cm} / \mathrm{s}(6 \%$ to $10 \%)$ for the velocity magnitude. For $\alpha$, it ranges from $4.3^{\circ}$ to $11^{\circ}\left(1 \%\right.$ to $3 \%$ ), and the range is $0.4^{\circ}$ to $6.6^{\circ}(0.2 \%$ to $4 \%)$ for $\beta$.

As illustrated by the results, it is possible to calculate the velocity magnitude and flow direction for the velocity vector at the center of the vessel.

\section{3-D Velocity Profiles}

The precision and accuracy for the estimated 3-D velocity vector at the center of the vessel was examined in the previous section. The following expands the performance analysis to the entire $3-\mathrm{D}$ velocity profiles.

For that purpose, the relative mean bias and standard deviation are employed as performance metrics [16], [32]. They calculate the mean bias or mean standard deviation over the entire profile. The velocities are then normalized by the peak velocity magnitude.

Table iI. Expected Velocity Magnitude and Flow Direction With Uncertainties at the Center of the Vessel Along With Mean, Standard Deviation, and Bias of Estimated Values

\begin{tabular}{|c|c|c|c|c|c|}
\hline \multirow[b]{2}{*}{ Metric } & \multicolumn{4}{|c|}{ Flow direction } & \multirow[b]{2}{*}{ Unit } \\
\hline & {$[0,0]^{\circ}$} & {$[45,0]^{\circ}$} & {$[45,15]^{\circ}$} & {$[45,45]^{\circ}$} & \\
\hline$|\mathbf{v}|$ & $50.0 \pm 0.25$ & $50.3 \pm 0.25$ & $50.1 \pm 0.25$ & $50.0 \pm 0.25$ & {$[\mathrm{~cm} / \mathrm{s}]$} \\
\hline$|\overline{\mathbf{v}}| \pm s_{|\mathbf{v}|}$ & $49.6 \pm 3.2$ & $52.7 \pm 5.1$ & $50.8 \pm 5.2$ & $48.2 \pm 5.2$ & {$[\mathrm{~cm} / \mathrm{s}]$} \\
\hline$B_{|\mathbf{v}|}$ & -0.36 & 2.4 & 0.71 & -1.8 & {$[\mathrm{~cm} / \mathrm{s}]$} \\
\hline$\alpha$ & $0 \pm 1$ & $45 \pm 1$ & $45 \pm 1$ & $45 \pm 1$ & {$\left[{ }^{\circ}\right]$} \\
\hline $\bar{\alpha} \pm s_{\alpha}$ & $-1.26 \pm 4.8$ & $45.4 \pm 4.3$ & $45.6 \pm 4.9$ & $46 \pm 11$ & {$\left[{ }^{\circ}\right]$} \\
\hline$B_{\alpha}$ & -1.3 & 0.45 & 0.6 & 1.1 & {$\left[{ }^{\circ}\right]$} \\
\hline$\underline{\beta}$ & $0 \pm 2$ & $0 \pm 2$ & $15 \pm 2$ & $45 \pm 2$ & {$\left[{ }^{\circ}\right]$} \\
\hline $\bar{\beta} \pm s_{\beta}$ & $2.0 \pm 0.52$ & $-1.42 \pm 0.40$ & $17.6 \pm 1.9$ & $47.7 \pm 6.6$ & {$\left[0^{\circ}\right]$} \\
\hline$B_{\beta}$ & 2.0 & -1.4 & 2.6 & 2.7 & {$\left[{ }^{\circ}\right]$} \\
\hline
\end{tabular}
For Four Different Directions. 
First, the mean and standard deviation of the 100 measured velocity profiles for the three estimated velocity components for all 16 different flow directions are presented [Dot-dashed lines indicate the expected profiles, solid lines indicate the mean of the profiles, and dashed lines indicate one standard deviation. The magnitudes of $v_{x}$ and $v_{y}$ depend on $\alpha$, whereas the magnitude of $v_{z}$ depends on $\beta$. As $\beta$ increases, so does the apparent width of the vessel because of the beam-to-flow angle.

Four of the flow directions are presented in Fig. 5. The figure demonstrates that the individual estimated velocity component profiles follow the expected profiles. In the left-most column, the intended flow direction was $(1,0,0)$, i.e., $[\alpha, \beta]=[0,0]^{\circ}$. This is, in fact, the case because both $v_{y}$ and $v_{z}$ are very close to $0 \mathrm{~m} / \mathrm{s}$. The mean values of the components are not exactly $0 \mathrm{~m} / \mathrm{s}$ because a perfect alignment with both angles being exactly $0^{\circ}$ is difficult to obtain in the measurement setup.
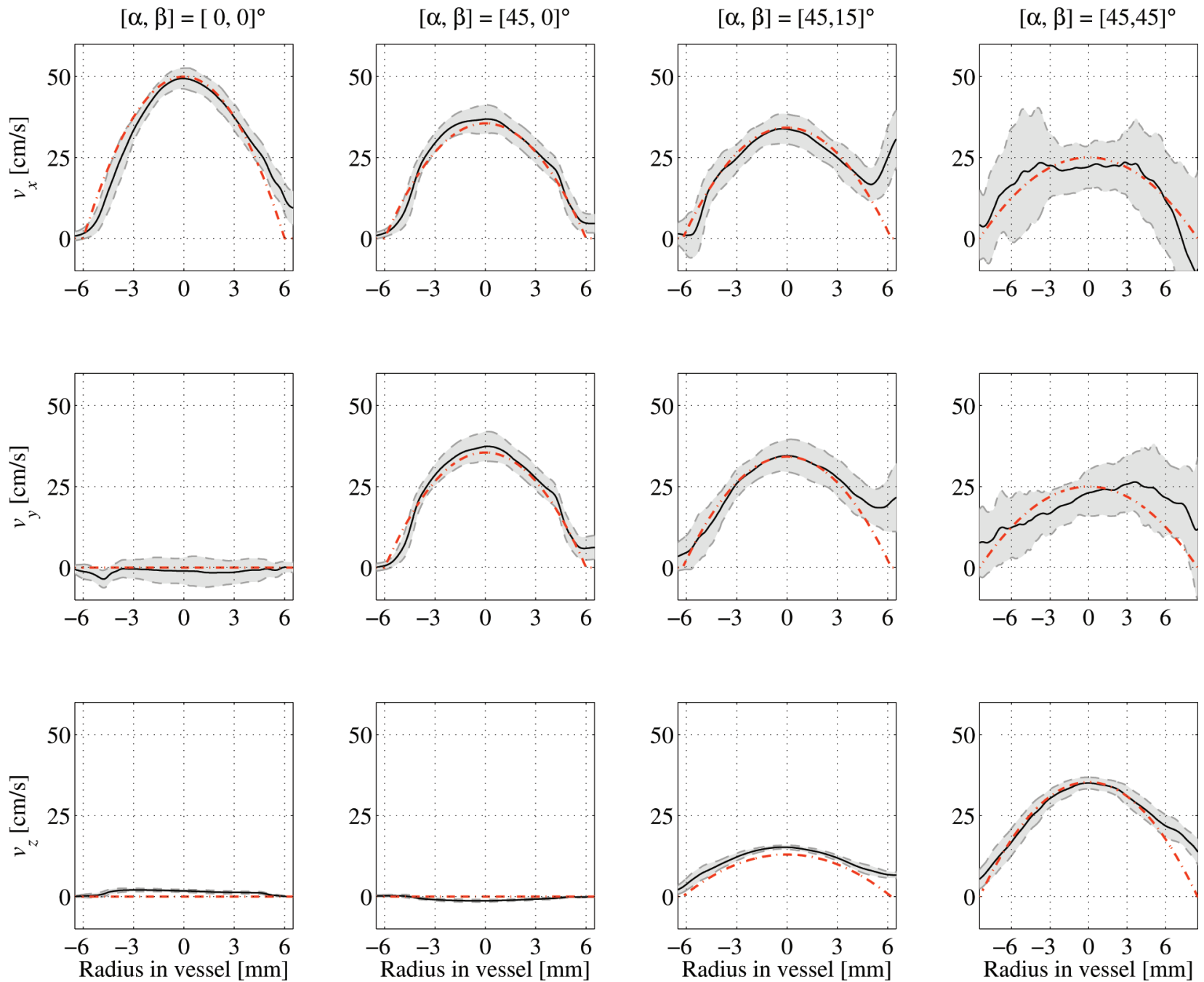

Fig. 5. Mean and standard deviation of the 100 measured velocity profiles for the three estimated velocity components (rows) for four different flow directions (columns). Dot-dashed lines indicate the expected profiles, solid lines indicate the mean of the profiles, and dashed lines one standard deviation. The magnitudes of $v_{x}$ and $v_{y}$ depend on $\alpha$, whereas the magnitude of $v_{z}$ depends on $\beta$. As $\beta$ increases, so does the apparent width of the vessel due to the beam-to-flow angle. 
in the companion paper [16]. The conclusion is that the pulse repetition frequency should be as low as possible. That is not the case for $v_{x}$ and $v_{y}$, where the components get farther from the maximum detectable velocity for increasing $\beta$.

Another observation is that the velocity estimates do not always reach $0 \mathrm{~m} / \mathrm{s}$ at the distal end of the wall (at positive radii). At times, the velocity components increase at the distal wall. This is caused by the strong reflections from the rubber tube resulting from the high impedance mismatch between the vessel wall and the surrounding fluids. That generates ringing, and therefore a ghost vessel below the real one. Then, because of the pulse length and the $\mathrm{RF}$ averaging, the $0 \mathrm{~m} / \mathrm{s}$ point within the distal vessel wall is smoothed out. The severity of this artifact depends on transducer orientation. Note, however, that this artifact is linked to the artificial nature of the setup, and would not be an issue in phantoms or in vivo, where the impedance mismatch is much smaller, and where stronger reflections from surrounding tissue would mask any remaining weak ghosting flow signal. For that reason, the performance metrics are calculated based on the central $10 \mathrm{~mm}$ of the vessel profiles.

The estimated velocity profiles presented in Fig. 5 are comparable to the simulation results obtained at the same flow directions, as reported in the accompanying paper [16]. For those results, the biases were also smaller than $4 \%$. The relative standard deviations ranged from $6 \%$ to $11 \%$ for $v_{x}$ and $v_{y}$ and were between $0.4 \%$ and $0.7 \%$ for $v_{z}$.

The performance metrics for the estimated velocity profiles are summarized in Fig. 6. Overall, the relative mean bias is within $\pm 5 \%$ for all three components. There are some repeated patterns in the relative biases for the different flow angles, which may reflect the fact that the measurements were conducted in several groups acquired at different times. As an example, the results for $\beta=0^{\circ}$ and $\alpha=[60,75,90]^{\circ}$ were acquired sequentially, followed by the data for $\beta=15^{\circ}$ and $\alpha=[90,75,60]^{\circ}$.

The relative standard deviations are consistent and independent of $\alpha$. For $\beta=0^{\circ}$, it is approximately $7 \%$ for $v_{x}$ and $v_{y}$ and $1 \%$ for $v_{z}$. When $\beta$ increases, the precision decreases as previously described.

Overall, the results demonstrate that the decoupling of the velocity estimation is successful, and that all three velocity components can be estimated simultaneously from the same data.

\section{Velocity Magnitude and Flow Angle Profiles}

The calculated magnitude and flow direction for the entire velocity profiles are presented in Fig. 7, which shows the resulting mean and standard deviation of $|\mathbf{v}|, \alpha$, and $\beta$ for the four cases presented in Fig. 5. The top row demonstrates that true velocity magnitude is estimated, albeit with decreasing precision as the magnitude of $\beta$ increases.

The next two rows demonstrate that the method estimates the correct direction throughout the main part of

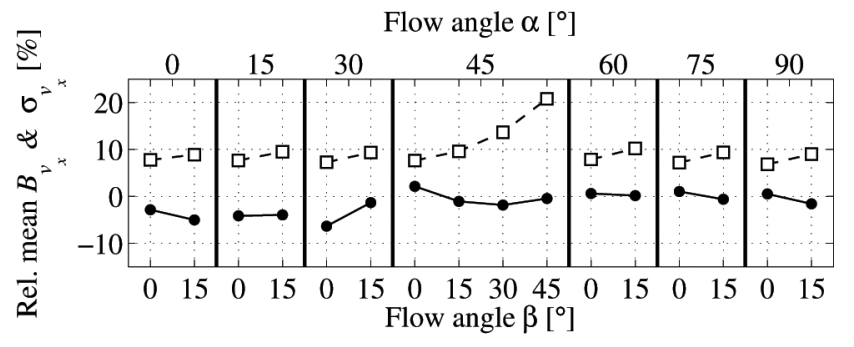

(a)

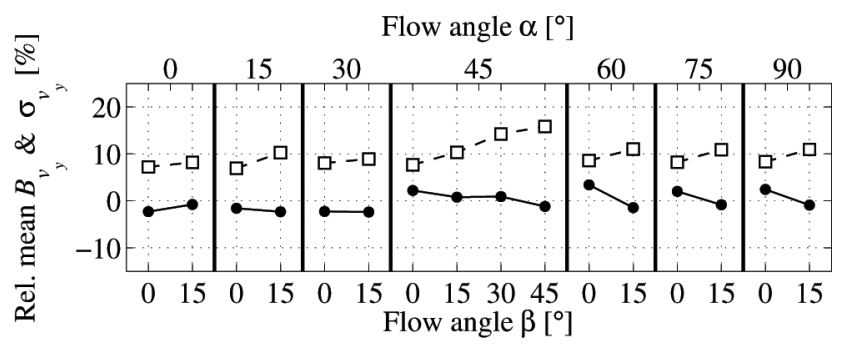

(b)

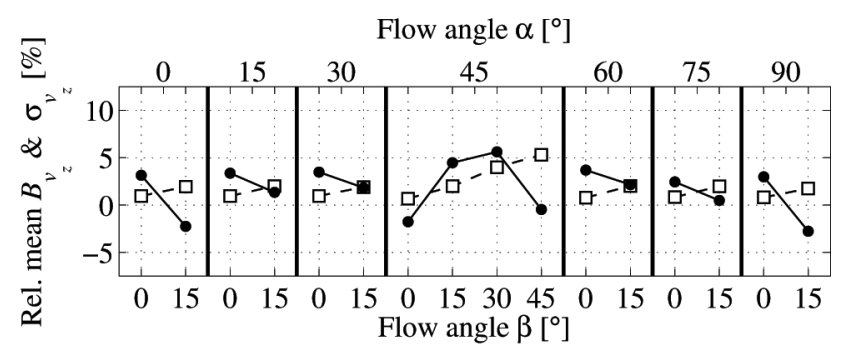

(c)

Fig. 6. The two performance metrics for (a) $v_{x}$, (b) $v_{y}$, and (c) $v_{z}$. The (relative) mean bias (dots) and (relative) mean standard deviations (squares) are shown for the 16 combinations of $\alpha$ (top axis) and $\beta$ (bottom axis).

the vessel. The uncertainty only increases at the boundaries because of the very small velocity components. Here, small fluctuations in the velocity estimates (particularly around $0 \mathrm{~m} / \mathrm{s}$ ) severely affect the estimated angles. It can be observed that the angular deviation is larger for $\alpha$ than for $\beta$. The reason is that $\alpha$ is estimated based on $v_{x}$ and $v_{y}$. These two components have a higher combined angular deviation compared with $v_{z}$ and $|\mathbf{v}|$. Consequently, the angular deviations increase as the standard deviations on the individual velocity components increase.

The performance metrics calculated over the entire profiles for four flow directions are listed in Table III. The values for all sixteen flow directions are shown in Fig. 8 .

The relative mean bias of the velocity magnitude is smaller than $\pm 6 \%$ for all directions and the standard deviations range from $8 \%$ to $16 \%$. On average for all directions, the relative mean bias is $-0.08 \%$. This emphasizes that the velocity estimation - when the TO method is properly optimized - is performed without bias.

For the flow angle $\alpha$, the average bias for all directions is $-0.2^{\circ}$ and the range of the angular deviations is $5^{\circ}$ to $16^{\circ}$. For $\beta$, the average bias is $-1.5^{\circ}$, and the angular 

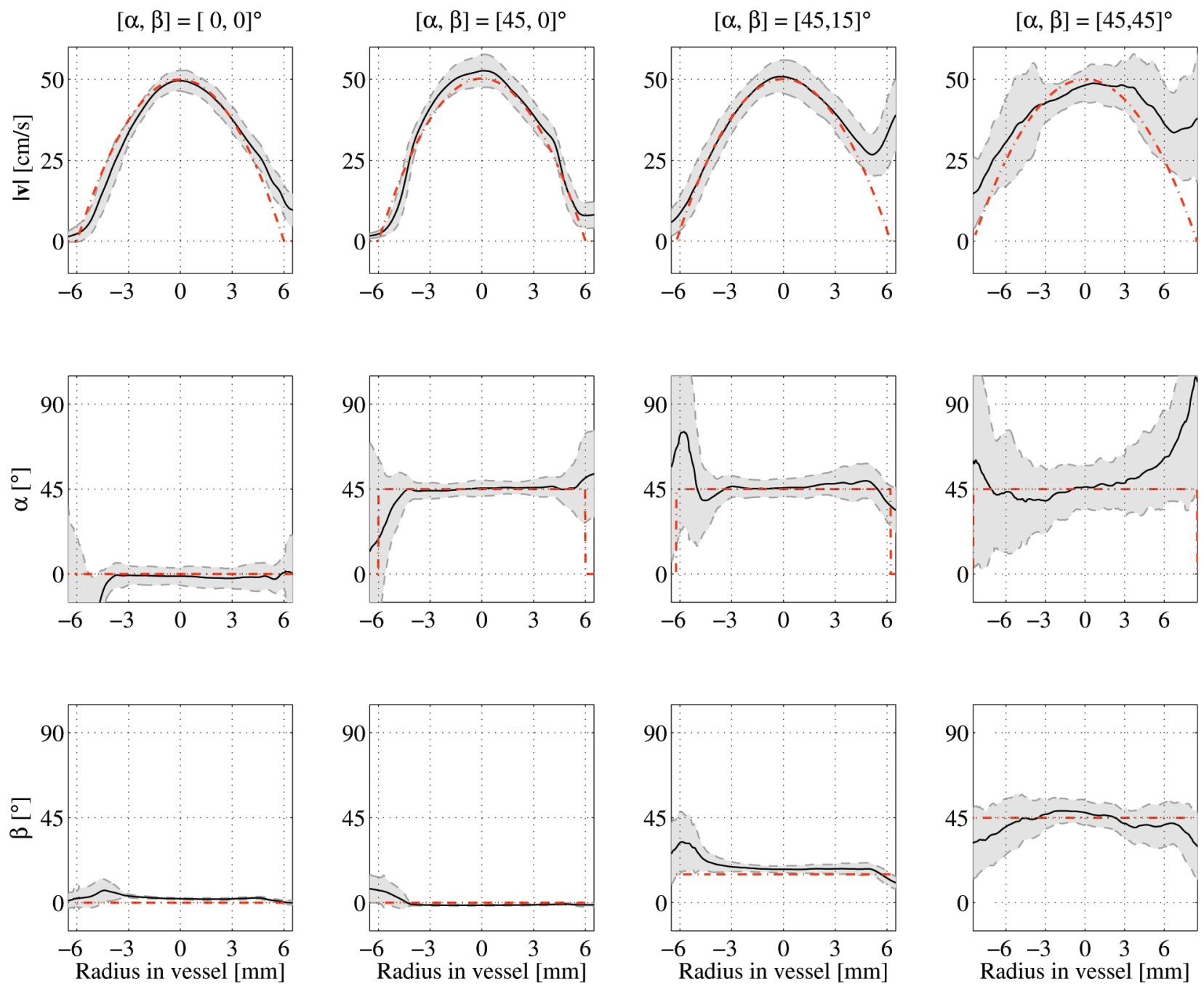

Fig. 7. The estimated velocity magnitude profiles and flow angles $\alpha$ and $\beta$. Mean values (solid) and the standard deviations or angular deviations (dashed) are indicated along with the expected values (dot-dashed). The four cases shown are the same as in Fig. 5.

deviations ranges from $0.7^{\circ}$ to $8^{\circ}$. Note that the angular deviations are less than $10^{\circ}$ and $3^{\circ}$ for $\alpha$ and $\beta$, when $\beta$ is $15^{\circ}$ and smaller. Most peripheral vessel will fall into this category.

The observed bias of $\beta$ is significantly different from $0^{\circ}$ for most of the flow directions. However, the biases are both positive and negative. The inaccuracies stem from alignment errors of the transducer. It should be noted that all the measurements with $\beta=0^{\circ}$ (except for $\alpha=45^{\circ}$ ) were conducted consecutively without adjusting transducer or fixture position except for the rotation of $\alpha$. This supports the notion that the observed systematic bias is a result of misalignment, and not a bias of the angle estimation itself.

The results demonstrate that the estimation of the flow angles is accurate, albeit with varying degree of precision depending on the pulse repetition frequency.

table iII. Mean Bias and Mean Standard Deviations of the Velocity Magnitude Profiles and Flow Angle Profiles.

\begin{tabular}{lrcccl}
\hline & \multicolumn{5}{c}{ Flow direction } \\
\cline { 2 - 4 } Metric & {$[0,0]^{\circ}$} & {$[45,0]^{\circ}$} & {$[45,15]^{\circ}$} & {$[45,45]^{\circ}$} & Unit \\
\hline$\tilde{B}_{|\mathbf{v}|}$ & -2.3 & 3.3 & 1.8 & 1.9 & {$[\%]$} \\
$\tilde{\sigma}_{|\mathbf{v}|}$ & 7.7 & 9.0 & 11 & 16 & {$[\%]$} \\
$\tilde{B}_{\alpha}$ & -2.5 & -0.16 & 0.88 & -0.90 & {$\left[{ }^{\circ}\right]$} \\
$\tilde{\sigma}_{\alpha}$ & 6.3 & 4.9 & 7.5 & 16 & {$\left[^{\circ}\right]$} \\
$\tilde{B}_{\beta}$ & -2.6 & 1.1 & -3.2 & 1.6 & {$\left[^{\circ}\right]$} \\
$\tilde{\sigma}_{\beta}$ & 1.4 & 0.75 & 2.8 & 8.0 & {$\left[{ }^{\circ}\right]$} \\
\hline
\end{tabular}




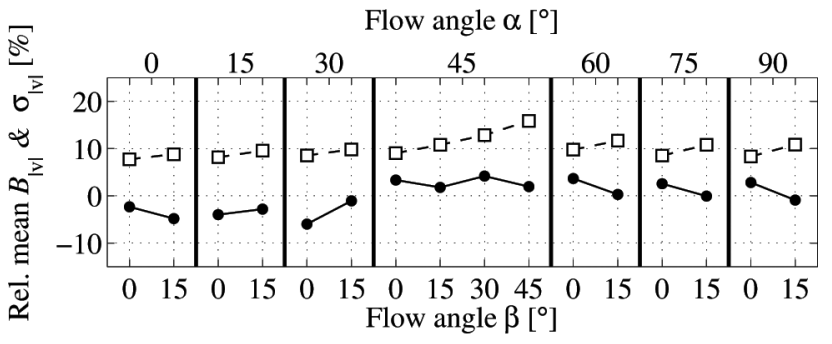

(a)

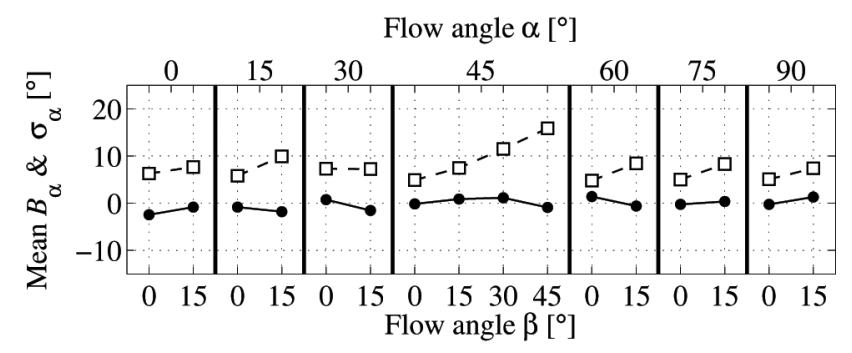

(b)

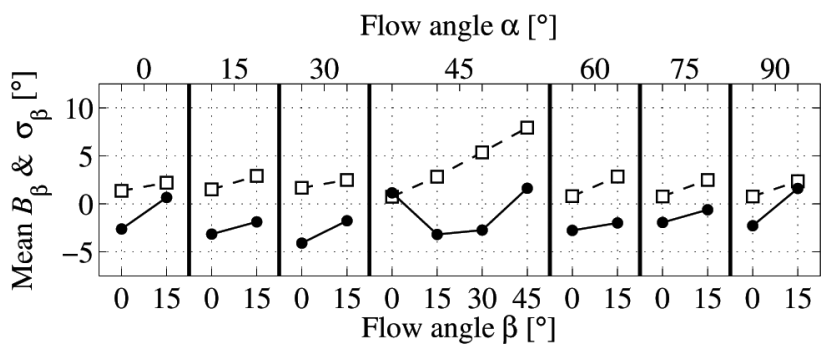

(c)

Fig. 8. The two performance metrics for (a) $|\mathbf{v}|$, (b) $\alpha$, and (c) $\beta$. The (relative) mean bias (dots) and (relative) mean standard deviations (squares) are shown for the 16 combination of $\alpha$ (top axis) and $\beta$ (bottom axis).

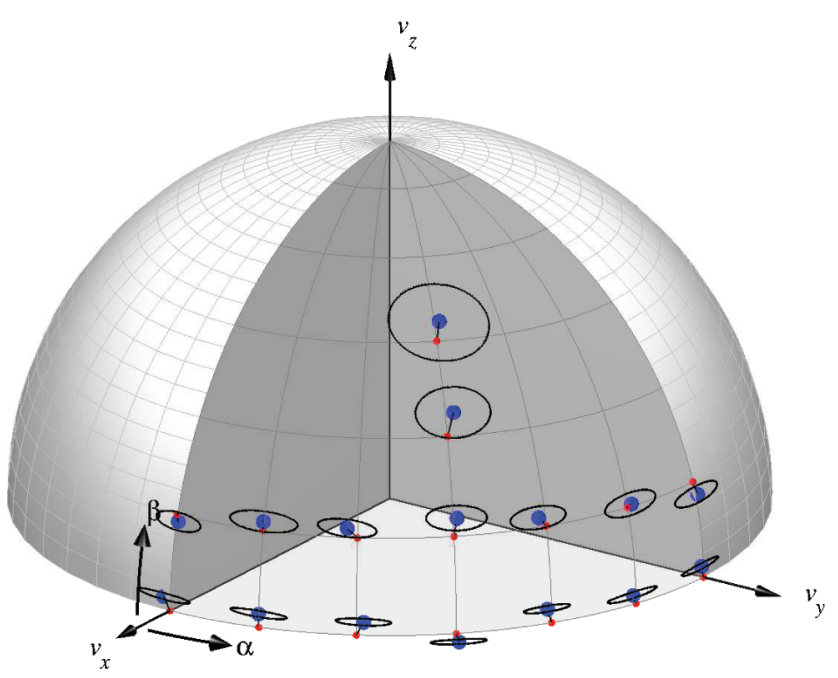

Fig. 9. Visualization of the estimated flow directions. Large dots indicating the mean direction are connected to small dots (if visible) indicating the expected flow direction. The magnitudes of the major and minor radii in the ellipses represent the standard deviations for those axes. The major axis is aligned with the direction of highest data density.

\section{E. Visualization of Mean Spherical Direction}

The results stated previously only considered $\alpha$ and $\beta$ separately. To investigate the mean estimated direction and take the full spherical nature of the flow directions into account, spherical statistics are employed.

Fig. 9 visualizes the mean flow direction for the sixteen measurements with the expected direction and the angular deviations. The figure illustrates that the estimated directions are practically as expected when taking the alignment inaccuracies on $\beta$ into account.

\section{CONCLUSION}

Through experimental measurements, this paper demonstrates that the 3-D transverse oscillation method using a 2-D transducer synthesizes two double-oscillating fields that oscillate in the axial direction and either the transverse or the elevation direction. The double oscillating fields combined with spatial quadrature sampling form the foundation for the 3-D TO method.

Three-dimensional velocity estimation is performed on experimental data acquired from a flow-rig system with steady flow. Measurements are conducted for sixteen different flow directions. The estimated profiles of the three spatial velocity components follow the expected values.

Compared with simulated results [16], the measurement results exhibit similar performance. The biases and the standard deviations on the velocity components are of comparable magnitude.

The estimation of the full 3-D velocity vectors allows for calculations of the true velocity magnitude and direction. Calculated over the entire flow profiles, the mean relative bias for all sixteen directions is merely $-0.08 \%$ normalized with the peak velocity magnitude. This demonstrates that when properly optimized, the method estimates the velocity magnitudes without bias. For the flow angles $\alpha$ and $\beta$, the mean biases are $-0.2^{\circ}$ and $-1.5^{\circ}$, respectively. The precision of the results depends on the pulse repetition frequency. The standard deviation of the velocity magnitude ranges from $8 \%$ to $16 \%$. The angular deviations are in the ranges $5^{\circ}$ to $16^{\circ}$ and $0.7^{\circ}$ to $8^{\circ}$ for $\alpha$ and $\beta$, respectively.

The flow rig measurements confirm that the 3-D transverse oscillation methods estimates the full three-dimensional velocity vectors with accurate velocity magnitude and directionality independent of the flow direction and, hence, transducer orientation.

Because the three velocity components are estimated simultaneously and from the same data set, it will be possible to measure and visualize the temporally changing complex flow patterns found throughout the circulatory system. In addition, estimation of true volumetric flow rates can be obtained by summing the out-of-plane velocity components in cross-sectional planes of vessels. 


\section{REFERENCES}

[1] S. Satomura, "Ultrasonic Doppler method for the inspection of cardiac functions," J. Acoust. Soc. Am., vol. 29, no. 11, pp. 1181-1185, 1957.

[2] P. J. Kilner, G. Z. Yang, R. H. Mohiaddin, D. N. Firmin, and D. B. Longmore, "Helical and retrograde secondary flow patterns in the aortic arch studied by three-directional magnetic resonance velocity mapping," Circulation, vol. 88, no. 5, pp. 2235-2247, 1993.

[3] C. Taylor, T. Hughes, and C. Zarins, "Finite element modeling of blood flow in arteries," Comput. Methods Appl. Mech. Eng., vol. 158, no. 1, pp. 155-196, 1998.

[4] K. L. Hansen, J. Udesen, F. Gran, J. A. Jensen, and M. B. Nielsen, "In-vivo examples of complex flow patterns with a fast vector velocity method," Ultraschall Med., vol. 30, no. 5, pp. 471-477, 2009

[5] R. E. Daigle, C. W. Miller, M. B. Histand, F. D. McLeod, and D. E. Hokanson, "Nontraumatic aortic blood flow sensing by use of an ultrasonic esophageal probe," J. Appl. Physiol., vol. 38, no. 6, pp. 1153-1160, 1975.

[6] M. D. Fox and W. M. Gardiner, "Three-dimensional Doppler velocimetry of flow jets," IEEE Trans. Biomed. Eng., vol. 35, no. 10, pp. 834-841, 1988

[7] B. Dunmire, K. W. Beach, K.-H. Labs, M. Plett, and D. E. Strandness, "Cross-beam vector Doppler ultrasound for angle independent velocity measurements," Ultrasound Med. Biol., vol. 26, no. 8, pp. 1213-1235, 2000.

[8] L. N. Bohs, B. J. Geiman, M. E. Anderson, S. C. Gebhart, and G. E. Trahey, "Speckle tracking for multi-dimensional flow estimation," Ultrasonics, vol. 38, no. 1-8, pp. 369-375, 2000

[9] A. A. Morsy and O. T. von Ramm, "FLASH correlation: A new method for 3-D ultrasound tissue motion tracking and blood velocity estimation," IEEE Trans. Ultrason. Ferroelectr. Freq. Control, vol. 46, no. 3, pp. 728-736, 1999.

[10] A. McArdle, V. Newhouse, and K. Beach, "Demonstration of threedimensional vector flow estimation using bandwidth and two transducers on a flow phantom," Ultrasound Med. Biol., vol. 21, no. 5, pp. 679-692, 1995

[11] J. M. Rubin, T. A. Tuthill, and J. B. Fowlkes, "Volume flow measurement using Doppler and grey-scale decorrelation," Ultrasound Med. Biol., vol. 27, no. 1, pp. 101-109, 2001.

[12] T. Tuthill, J. Rubin, and J. Fowlkes, "Three-dimensional flow vectors from RF ultrasound signals," in Proc. SPIE Medical Imaging, 2002, vol. 4687, pp. 210-217.

[13] O. Bonnefous, "Measurement of the complete (3D) velocity vector of blood flows," in Proc. IEEE Ultrasonics Symp., 1988, pp. 795-799.

[14] I. A. Hein, "3-D flow velocity vector estimation with a triple-beam lens transducer-Experimental results," IEEE Trans. Ultrason. Ferroelectr. Freq. Control, vol. 44, no. 1, pp. 85-95, 1997.

[15] C. Poelma, J. M. Mari, N. Foin, M. Tang, R. Krams, C. G. Caro, P. D. Weinberg, and J. Westerweel, "3D flow reconstruction using ultrasound PIV," Exp. Fluids, vol. 50, no. 4, pp. 777-785, 2011.

[16] M. J. Pihl and J. A. Jensen, "A transverse oscillation approach for estimation of three-dimensional velocity vectors. Part I: Concept and simulation study," IEEE Trans. Ultrason. Ferroelectr. Freq. Control, vol. 61, no. 10, pp. 1599-1607, 2014.

17] J. Udesen, M. B. Nielsen, K. R. Nielsen, and J. A. Jensen, "Examples of in-vivo blood vector velocity estimation," Ultrasound Med. Biol., vol. 33, no. 4, pp. 541-548, 2007.

[18] P. M. Hansen, M. M. Pedersen, K. L. Hansen, M. B. Nielsen, and J. A. Jensen, "Demonstration of a vector velocity technique," Ultraschall Med., vol. 32, no. 2, pp. 213-215, 2011.

[19] K. L. Hansen, M. M. Pedersen, H. Møller-Sørensen, J. Kjaergaard, J. C. Nilsson, J. T. Lund, J. A. Jensen, and M. B. Nielsen, "Intraoperative cardiac ultrasound examination using vector flow imaging," Ultrason. Imaging, vol. 35, no. 4, pp. 318-332, Oct. 2013.

[20] N. I. Fisher, T. Lewis, and B. J. J. Embleton, Statistical Analysis of Spherical Data. New York, NY: Cambridge University Press, 1987.

[21] P. Berens, "Circstat: A MATLAB toolbox for circular statistics," J. Stat. Softw., vol. 31, no. 10, pp. 1-21, 2009.

[22] P. Leong and S. Carlile, "Methods for spherical data analysis and visualization," J. Neurosci. Methods, vol. 80, no. 2, pp. 191-200, 1998.

[23] L. Ratsimandresy, P. Mauchamp, D. Dinet, N. Felix, and R. Dufait, "A $3 \mathrm{MHz}$ two dimensional array based on piezocomposite for medical imaging," in Proc. IEEE Ultrasonics Symp., 2002, pp. 1265-1268.
[24] L. Ratsimandresy, N. Felix, D. Dinet, and R. Dufait, "2D arrays performances optimization to address high quality volumetric imaging," in Proc. IEEE Ultrasonics Symp., 2005, pp. 657-660.

[25] J. A. Jensen, H. Holten-Lund, R. T. Nielson, B. G. Tomov, M. B. Stuart, S. I. Nikolov, M. Hansen, and U. D. Larsen, "Performance of SARUS: A synthetic aperture real-time ultrasound system," in Proc. IEEE Ultrasonics Symp., Oct. 2010, pp. 305-309.

[26] K. V. Ramnarine, D. K. Nassiri, P. R. Hoskins, and J. Lubbers, "Validation of a new blood mimicking fluid for use in Doppler flow test objects," Ultrasound Med. Biol., vol. 24, no. 3, pp. 451-459, 1998.

[27] J. M. Hansen, M. C. Hemmsen, and J. A. Jensen, "An object-oriented multi-threaded software beamformation toolbox." in Proc. SPIE Medical Imaging, 2011, vol. 7968, art. no. 79680Y.

[28] J. A. Jensen, "A new estimator for vector velocity estimation," IEEE Trans. Ultrason. Ferroelectr. Freq. Control, vol. 48, no. 4, pp. 886-894, 2001.

[29] J. A. Jensen, "Field: A program for simulating ultrasound systems," Med. Biol. Eng. Comput., vol. 34, suppl. 1, pt. 1, pp. 351-353, 1996.

[30] J. A. Jensen and N. B. Svendsen, "Calculation of pressure fields from arbitrarily shaped, apodized, and excited ultrasound transducers," IEEE Trans. Ultrason. Ferroelectr. Freq. Control, vol. 39, no. 2, pp. 262-267, 1992.

[31] M. J. Pihl and J. A. Jensen, "Measuring 3D velocity vectors using the transverse oscillation method," in Proc. IEEE Ultrason. Symp., 2012, pp. 1881-1885

[32] M. J. Pihl, J. Marcher, and J. A. Jensen, "Phased-array vector velocity estimation using transverse oscillations," IEEE Trans. Ultrason. Ferroelectr. Freq. Control, vol. 59, no. 12, pp. 2662-2675, 2012.

[33] J. A. Jensen, Estimation of Blood Velocities Using Ultrasound: A Signal Processing Approach. New York, NY: Cambridge University Press, 1996.

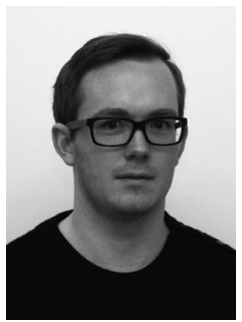

Michael J. Pihl was born in 1983. He received his M.Sc. degree in biomedical engineering in 2009 from the Technical University of Denmark (DTU) and the University of Copenhagen, Denmark. In 2007, he spent seven months at the Biomedical Engineering Department at Duke University, Durham, NC, researching ultrasonic clutter. In 2012, he earned his $\mathrm{Ph} . \mathrm{D}$. degree in biomedical engineering at the Center for Fast Ultrasound Imaging at DTU, where he is currently a postdoctoral associate. His main research area is ultrasonic velocity estimation - in particular the estimation of all three spatial velocity components. His other interests include medical imaging and digital signal processing.

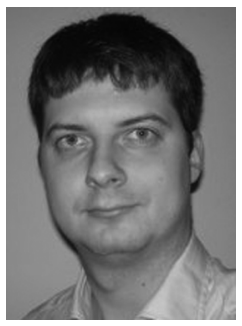

Matthias Stuart received the M.Sc. and Ph.D. degrees in Computer Engineering in 2006 and 2010 respectively, both from the Technical University of Denmark, Lyngby, Denmark, in 2006. He is currently a postdoc with the Center for Fast Ultrasound Imaging at the Department of Electrical Engineering, Technical University of Denmark. His research interests include synthetic aperture methods for both anatomical and flow imaging, ultrasound systems, and real-time implementations of ultrasound processing algorithms.

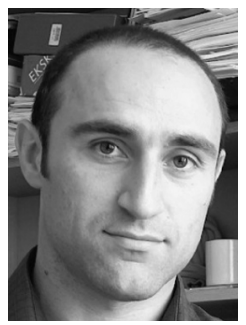

Borislav G. Tomov obtained his M. Sc. degree in Electronics Engineering from the Technical University of Sofia, Bulgaria, in 1996, and his $\mathrm{Ph} . \mathrm{D}$. degree in Medical Electronics from the Technical University of Denmark in 2003. His research interests include medical ultrasound signal processing, and ultrasound scanner architectures and implementations. 


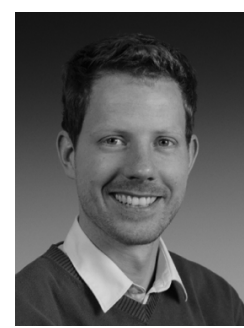

Morten F. Rasmussen was born in 1981. He received a B.Sc. degree and a M.Sc. degree in electrical engineering from the Technical University of Denmark (DTU), in 2008 and 2011, respectively. In 2007 he spent ten months at the Technische Universität Berlin, Berlin, Germany, working on the BeeSat satellite. He is currently pursuing his Ph.D. degree in electrical and biomedical engineering at the Center for Fast Ultrasound Imaging at DTU. The topics of his Ph.D. thesis are 3-D ultrasound imaging and synthetic aperture imaging techniques.

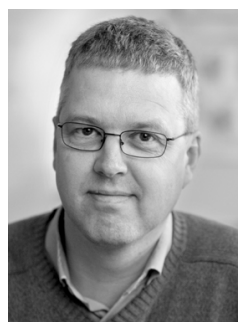

Jørgen Arendt Jensen earned his Master of Science degree in electrical engineering in 1985 and the Ph.D. degree in 1989, both from the Technical University of Denmark. He received the Dr.Techn. degree from the university in 1996. He has published more than 250 journal and conference papers on signal processing and medical ultrasound and the book Estimation of Blood Velocities Using Ultrasound, published by Cambridge University Press in 1996. He is also developer of the Field II simulation program. He has been a visiting scientist at Duke University, Stanford University, and the University of Illinois at Urbana-Champaign. He is currently full professor of Biomedical Signal Processing at the Technical University of Denmark at the Department of Electrical Engineering and head of the Center for Fast Ultrasound Imaging. He has given courses on blood velocity estimation at both Duke University and the University of Illinois and teaches biomedical signal processing and medical imaging at the Technical University of Denmark. He has given several short courses on simulation, synthetic aperture imaging, and flow estimation at international scientific conferences. He has received several awards for his research and is an IEEE Fellow. His research is centered on simulation of ultrasound imaging, synthetic aperture imaging, vector blood flow estimation, and construction of ultrasound research systems. 